dissemination of Gaussian-based "significance" tests.

If or when the percentile method of expressing interindividual differences has been widely adopted, there will still remain two much more difficult problems: (a) the collection, from healthy and diseased subjects, of data that are reliable and are applicable to the particular patient under investigation; and (b) the question of where to make the cut-off points between "normal" and "abnormal"what risks of error (false-positives and falsenegatives) are acceptable. For the balancirg of these risks-often a different balance with respect to different disorders, different consequences of error, and even different patients -we must look to clinicians, not to biochemists or other laboratory workers, or to statisticians as such.

It would be interesting if the SI units compensated somewhat for their adverse effects by promoting the use of a sensible method of expressing intermeasurement variation and by allowing us to focus attention on how to collect data and apply them to individual patients.

Donald Mainland Kent,
Conn, USA

'Mainland, D, Elementary Medical Statistics, 2nd edn, p 265. Philadelphia, W B Saunders Company, 1963. peutics, 1974, 15, 528 .

Elveback, L R, et al, fournal of the American Medical Association, 1970, 211, 69

Mainland, D, Clinical Chemistry, 1971, 17, 267.

isher, R A, Philosophical Transactions of the Royal

Society of London, A, 1921, 222, 309

Edinburgh, Oliver and Boyd, 1925.
isher, R A, Statich Workers.

\section{Surgical treatment of coronary disease}

SIR,- - The Veterans Administration co-operative study of the surgical treatment of coronary artery disease represents probably the most valuable contribution to an understanding of the role of surgery that is available at present. Minerva (15 October, p 1032) therefore does a grave disservice in misquoting the conclusions of a recent interim report from that study. ${ }^{1}$ This showed no difference in mortality between the medically and surgically treated groups over a three-year period. However, the prime indication for surgery is angina pectoris and the prime objective is the relief of this symptom. By reporting the study as showing that "patients treated by bypass for chronic stable angina had done no better than those given beta blockers" Minerva implies that pain relief was not achieved. In fact, this aspect was not included in the report, although elsewhere in the same journal Braunwald states that coronary artery bypass grafting reduces the incidence and severity of angina. ${ }^{2}$

The failure to detect a difference in survival between the two groups may reflect a true failure of surgery to improve prognosis, but in view of the high graft occlusion rate $(12 \%$ of the patients had no patent grafts at restudy) it could also point to the need to develop techniques whereby graft occlusion can be minimised. No analysis was made of the relation between graft patency and death, and since $56 \%$ of deaths in the surgical group occurred in the first 30 days the status of the grafts in most of the surgical deaths is not known.

The Veterans Administration study surely makes three points abundantly clear. Firstly, the prognosis is related to the distribution of the coronary artery lesions, which can be assessed only by angiography. ${ }^{3}$ Secondly, many problems remain to be solved in relation to the intraoperative and postoperative management of patients treated surgically to improve graft patency. Thirdly, the relation between graft patency and survival is not yet known. This in no way detracts from the fundamental point, which is that coronary bypass surgery is an effective method of alleviating angina pectoris and at the very least does not diminish longevity. Surely the relief of an often incapacitating symptom is valuable in itself.

London Chest Hospital.

Clive a layton London E2

Murphy, M L, et al, New England fournal of Medicine, 1977, 297, 621.
Braunwald, E, New England Fournal of Medicine, 1977, 297, 66

Hultgren, H N, Takaro, T, and Detre, K, Circulation, 1976, suppl II, p 172 .

\section{Hyperglycaemia and complications of diabetes}

SIR,-One of us (RR) has recently found that maintenance of a normal blood glucose concentration in streptozotocin-diabetic rats prevents the development of basement membrane thickening in the renal glomeruli. ${ }^{1}$

Dr C J Fox and others (3 September, p 605) studied "the effects of diabetic control on basement membrane thickening in rats" and concluded that there is a "strong relation between basement membrane thickening and hyperglycaemia." They add that "these findings should offer encouragement not only to those who treat diabetic patients with the aim of maintaining good blood glucose control but also to those who search for improved methods of achieving this aim."

Unfortunately, however, the above conclusion cannot be drawn from their results. It is based on a regression analysis including the non-diabetic rats, a procedure which, of course, is quite unacceptable. The lack of relation between hyperglycaemia and basement membrane thickness is revealed by the values obtained in the four groups of diabetic rats. It appears that there were no consistent differences in basement membrane thickness between diabetic groups with average plasma glucose levels of $9 \cdot 8,19 \cdot 8,21 \cdot 5$, and $27 \cdot 4$ $\mathrm{mmol} / 1(176,356,387$, and $493 \mathrm{mg} / 100 \mathrm{ml})$ respectively.

There are other unacceptable features in their paper, but it is not necessary to discuss them here.

\section{Second University Clinic
of Internal Medicine,}

HaNS J JRGEN G GUNDERSEN

Institute of Experimental

Clinical Research,

Denmark

Rasch, R, Diabetologia, 1977, 13, 426

${ }^{*}{ }^{*}$ The authors sent a copy of this letter to Dr Fox and his colleagues, whose reply is printed below.-ED, $B M \mathcal{A}$.

SIR,-Measurements of hyperglycaemia and basement membrane thickness, like most biological data (for example, blood pressure and serum cholesterol), form a continuum from normal through slightly deranged to grossly abnormal. To establish the relationship between these two variables it is necessary to look at both ends of the spectrum, and of course data from non-diabetics have to be included in the analysis.

If streptozotocin per se were to affect the basement membrane, then results from diabetic rats could not be grouped together with those from non-diabetic controls. That this is not the case has been shown by Dr Rasch herself. Thus in the paper quoted above streptozotocindiabetic rats carefully controlled on insulin for six months have no increase in glomerular basement membrane thickness over normal controls.

Charles Fox

$S$ C DARBY

P H SÖNKSEN

Department of Medicine,

London SE

Medical Unit B

Medical Unit B,
Southern General Hospital,

Glasgow

JOHN T IRELAND

\section{Control of hyperglycaemia in young} diabetics

SIR,-Recent reports ${ }^{1-3}$ have indicated that good control of hyperglycaemia may decrease the microvascular complications of diabetes mellitus.

At a recent summer camp for adolescent diabetics an opportunity arose to determine the views of the children on the control of their diabetes. Eighteen diabetics (mean age 15! years) from most areas of England attended the camp; all were insulin-dependent and the mean duration of disease was 8 years. Only nine were being treated with a twice-daily regimen of soluble insulin together with an intermediate-action insulin, a regimen which many diabetologists would consider essential for good control of hyperglycaemia. Three of the group were being treated with a morning injection of Monotard insulin ranging from 20 to $80 \mathrm{U}$ /day.

Ten of the patients performed urine tests on two or more occasions each day, but six children tested only once each day or less; the remaining two patients had low renal thresholds and so did not rely on urine testing. Only two of the group accepted urine tests showing no sugar as good control and four patients were satisfied with mainly negative reactions with occasional $\frac{1}{4} \%$ sugar, while 10 of the children preferred persistent glycosuria in their urine tests, five maintaining urine tests showing $\frac{1}{2}-\frac{3}{4} \%$ sugar. One of the patients with low renal threshold used Dextrostix and maintained her blood sugar between 10 and $13 \mathrm{mmol} / 1$ (180 and $240 \mathrm{mg} / 100 \mathrm{ml}$ ); the other patient did not use Dextrostix and did not test her urine.

Only seven of the group said that they would be happy if all their urine tests showed no sugar. Six patients would decrease their insulin dosage in this situation and a further three would take more carbohydrate exchanges. It is distressing that seven of the children (excluding the two with low renal threshold) felt that it was impossible to have continuous negative urine tests without frequent hypoglycaemic attacks. Despite the above findings 17 out of the 18 patients felt that good control was important for their future wellbeing and it was encouraging that seven of the group felt that the amount of animal fat in their diet might be of importance, six of 